Manuscript accepted for publication as a Regular Article in Phys. Rev. B

\title{
Numerical computation of the response of piezoelectric composites using Fourier transform
}

\author{
R. Brenner \\ Laboratoire des Propriétés Mécaniques et Thermodynamiques des Matériaux, \\ CNRS, Université Paris Nord, 93430 Villetaneuse, France*
}

(Dated: April 13, 2009)

\begin{abstract}
An efficient numerical scheme is presented to compute the response of piezoelectric composite materials with arbitrary complex microstructures. It makes use of Fast Fourier Transform (FFT) to solve iteratively the coupled periodic Lippmann-Schwinger equations for an heterogeneous electroelastic medium. The method is assessed in the case of a two-phase composite by comparison with analytic solutions and finite-element results taken from the literature.
\end{abstract}

PACS numbers: 46.25.Hf, 47.11.Kb

Keywords: composite, piezoelectric, Fourier transform, periodic homogenization 


\section{INTRODUCTION}

This work aims at describing an efficient numerical scheme to determine the overall behaviour and the local fields within an heterogeneous medium presenting a coupled constitutive behaviour. The method is an extension of a computational approach initially proposed for elastic-type composites ${ }^{1}$. Up to now, numerical homogenization in the framework of coupled behaviours (e.g piezoelectric, piezomagnetic, thermoelastic etc.) has been essentially performed with the Finite-Element method $(\mathrm{FEM})^{2-5}$ which requires tedious meshing as soon as the microstructure becomes complex. The alternative method presented in this article has been previously used for uncoupled (linear and nonlinear) mechanical behaviours with various microstructures ${ }^{1,6-8}$ as well as other uncoupled physical properties ${ }^{9,10}$. It makes use of Fast Fourier Transform (FFT) and permits to use images of the microstructure for the computation.

Section II is devoted to the mathematical formulation of the problem to be solved. It is shown that the local fields are solutions of a set of coupled Lippmann-Schwinger (LS) equations. The iterative scheme which allows to solve the LS equations is detailed in section III. Results are presented for a two-phase composite material with laminate or fibrous microstructure in section IV.

\section{INTEGRAL EQUATIONS FOR HETEROGENEOUS ELECTROELASTICITY}

We consider a Representative Volume Element (RVE) $\Omega$ of a heterogeneous electroelastic material under isothermal conditions. The local properties of the material are thus defined by the elastic $\boldsymbol{C}$, the dielectric (permittivity) $\boldsymbol{\gamma}$ and the piezoelectric $\boldsymbol{e}$ moduli tensors. Relevant field variables to describe the behaviour of the medium are thus the elastic strain $\boldsymbol{\varepsilon}$, the stress $\boldsymbol{\sigma}$, the electric induction $\boldsymbol{D}$ and the electric field $\boldsymbol{E}$. The elastic strain and the electric field are respectively derivable from the displacement field $\boldsymbol{u}$ and the electric potential $\phi$. Tensor rank and units of the different variables of the problem are summarized in Table I.

To solve the problem, periodic boundary conditions are chosen and the RVE is considered as a unit cell. Using periodicity conditions stated by Suquet ${ }^{11}$, the fields $\boldsymbol{u}$ and $\phi$ on the 
TABLE I: Symbols and units

\begin{tabular}{lccc}
\hline \hline Physical quantity & Symbol & Tensor rank & Units \\
\hline Stress & $\boldsymbol{\sigma}$ & 2 & $\mathrm{~N} \mathrm{~m}^{-2}$ \\
Elastic strain & $\boldsymbol{u}$ & 2 & - \\
Elastic displacement & $\boldsymbol{D}$ & 1 & $\mathrm{~m}$ \\
Electric induction & $\boldsymbol{E}$ & 1 & $\mathrm{C} \mathrm{m}^{-2}$ \\
Electric field & $\phi$ & 0 & $\mathrm{~V} \mathrm{~m}^{-1}$ \\
Electric potential & $\boldsymbol{e}$ & 3 & $\mathrm{~V}$ \\
Piezoelectric moduli & $\boldsymbol{C}$ & 2 & $\mathrm{C} \mathrm{m}^{-2}$ \\
Permittivity moduli & & 4 & $\mathrm{~F} \mathrm{~m}^{-1}$ \\
Elastic moduli & & & $\mathrm{N} \mathrm{m}^{-2}$ \\
\hline \hline
\end{tabular}

boundary $\partial \Omega$ of the unit cell are decomposed into

$$
\boldsymbol{u}(\boldsymbol{x})=\overline{\boldsymbol{\varepsilon}} \cdot \boldsymbol{x}+\boldsymbol{u}^{\star}(\boldsymbol{x}) \text { and } \phi(\boldsymbol{x})=-\overline{\boldsymbol{E}} \cdot \boldsymbol{x}+\phi^{\star}(\boldsymbol{x})
$$

with $\overline{\boldsymbol{\varepsilon}}$ and $\overline{\boldsymbol{E}}$ prescribed average values of the strain and electric fields over $\Omega$. $\boldsymbol{u}^{\star}$ and $\phi^{\star}$ are periodic (\#) fluctuating terms. Besides, the mechanical and electric static equilibriums between neighboring cells imply anti-periodic (-\#) conditions for the traction vector $\boldsymbol{\sigma} . \boldsymbol{n}$ and the electric charge D. $\boldsymbol{n}$ on $\partial \Omega$ ( $\boldsymbol{n}$ is the outer unit normal on the boundary).

The problem to be solved can thus be expressed as: find $\boldsymbol{u}(\boldsymbol{x})$ and $\phi(\boldsymbol{x}), \forall \boldsymbol{x} \in \Omega$, such that

$$
\begin{cases}\text { Potential fields: } & \boldsymbol{\varepsilon}=\frac{1}{2}\left(\operatorname{grad} \boldsymbol{u}+{ }^{\mathrm{T}} \operatorname{grad} \boldsymbol{u}\right), \quad \boldsymbol{E}=-\operatorname{grad} \phi \\ \text { Local laws: } & \boldsymbol{\sigma}(\boldsymbol{x})=\boldsymbol{C}(\boldsymbol{x}): \boldsymbol{\varepsilon}(\boldsymbol{x})-{ }^{\mathrm{T}} \boldsymbol{e}(\boldsymbol{x}) \cdot \boldsymbol{E}(\boldsymbol{x}) \\ & \boldsymbol{D}(\boldsymbol{x})=\boldsymbol{e}(\boldsymbol{x}): \boldsymbol{\varepsilon}(\boldsymbol{x})+\boldsymbol{\gamma}(\boldsymbol{x}) \cdot \boldsymbol{E}(\boldsymbol{x}) \\ \text { Static equilibrium: } & \operatorname{div} \boldsymbol{\sigma}=0, \operatorname{div} \boldsymbol{D}=0 \\ \text { Boundary conditions: } & \boldsymbol{u}^{\star}, \phi^{\star} \# \text { and } \boldsymbol{\sigma} \cdot \boldsymbol{n}, \boldsymbol{D} \cdot \boldsymbol{n}-\#, \forall \boldsymbol{x} \in \partial \Omega .\end{cases}
$$

Note that the transpose of $\boldsymbol{e}$ is defined by ${ }^{\mathrm{T}} e_{i j n}=e_{n i j}$. The notation introduced by Barnett and Lothe ${ }^{12}$ to treat electroelastic problems is unnecessary for our study. Simple tensorial relations are kept to describe the problem.

By introducing a homogeneous reference medium with elastic $C^{0}$, permittivity $\gamma^{0}$ and piezoelectric $\boldsymbol{e}^{0}$ moduli tensors, the heterogeneous electroelastic problem is transformed into 
a homogeneous electroelastic problem with heterogeneous fields of fictitious body forces $\left(\boldsymbol{f}^{\prime}\right)$ and free electric charges $\left(q^{\prime}\right)^{13,14}$. Static equilibrium relations thus read

$$
\operatorname{div}\left(\boldsymbol{C}^{0}: \operatorname{grad} \boldsymbol{u}+{ }^{\mathrm{T}} \boldsymbol{e}^{0} \cdot \operatorname{grad} \phi\right)+\boldsymbol{f}^{\prime}=0 \quad \text { and } \quad \operatorname{div}\left(\boldsymbol{e}^{0}: \operatorname{grad} \boldsymbol{u}-\boldsymbol{\gamma}^{0} \cdot \operatorname{grad} \phi\right)+q^{\prime}=0
$$

with

$$
\boldsymbol{f}^{\prime}=\operatorname{div}\left(\delta \boldsymbol{C}: \operatorname{grad} \boldsymbol{u}+{ }^{\mathrm{T}} \delta \boldsymbol{e} \cdot \operatorname{grad} \phi\right), \quad q^{\prime}=\operatorname{div}(\delta \boldsymbol{e}: \operatorname{grad} \boldsymbol{u}-\delta \boldsymbol{\gamma} \cdot \operatorname{grad} \phi)
$$

The notation $\delta \boldsymbol{a}$ means $\boldsymbol{a}(\boldsymbol{x})-\boldsymbol{a}^{0}$. The system of differential equations (3) can be solved using standard Green's functions method. In linear piezoelectric media, the electroelastic Green's functions describe the elastic displacement $\boldsymbol{u}$ and electric potential $\phi$ at point $\boldsymbol{x}$ due to a unit point force or electric charge at point $\boldsymbol{x}^{\prime}$. The solution to this coupled-field problem was derived by Deeg ${ }^{15}$. It has been used to obtain a generalization of Eshelby's inclusion solution in elasticity ${ }^{16,17}$ and to extend different micromechanical models to coupled electroelasticity $^{17-19}$. By making use of the superposition principle, Deeg's result allows to get the solution of the periodic homogeneous electroelastic problem (3) with fluctuating fields $\boldsymbol{f}^{\prime}(\boldsymbol{x})$ and $q^{\prime}(\boldsymbol{x})$. It is expressible as coupled periodic Lippmann-Schwinger equations

$$
\begin{gathered}
\boldsymbol{\varepsilon}(\boldsymbol{x})=\overline{\boldsymbol{\varepsilon}}-\boldsymbol{\Gamma}^{0} * \boldsymbol{\tau}(\boldsymbol{x})-{ }^{\mathrm{T}} \boldsymbol{\Upsilon}^{0} * \boldsymbol{P}(\boldsymbol{x}), \forall \boldsymbol{x} \in \Omega, \\
\boldsymbol{E}(\boldsymbol{x})=\overline{\boldsymbol{E}}+\boldsymbol{\Upsilon}^{0} * \boldsymbol{\tau}(\boldsymbol{x})+\boldsymbol{\Delta}^{0} * \boldsymbol{P}(\boldsymbol{x}), \forall \boldsymbol{x} \in \Omega
\end{gathered}
$$

which are linear integral equations for the fields $\varepsilon$ and $\boldsymbol{E}$ (the notation $*$ indicates a convolution product). $\boldsymbol{\tau}$ and $\boldsymbol{P}$ are polarization fields from which $\boldsymbol{f}^{\prime}$ and $q^{\prime}$ are respectively derivable. They read

$$
\boldsymbol{\tau}=\delta \boldsymbol{C}: \boldsymbol{\varepsilon}-{ }^{\mathrm{T}} \delta \boldsymbol{e} . \boldsymbol{E} \text { and } \boldsymbol{P}=\delta \boldsymbol{e}: \boldsymbol{\varepsilon}+\delta \boldsymbol{\gamma} . \boldsymbol{E} .
$$

It can be remarked that only three Green's operators $\Gamma^{0}, \Delta^{0}$ and $\Upsilon^{0}$ have to be introduced because of the symmetry of the piezoelectric effect (i.e the value of the strain $\boldsymbol{\varepsilon}$ at point $\boldsymbol{x}$ due to a unit negative electric induction at point $\boldsymbol{x}^{\prime}$ is equal to the value of the electric field $\boldsymbol{E}$ at point $\boldsymbol{x}$ due to a unit stress at point $\boldsymbol{x}^{\prime}$ ). The coupled periodic Lippmann-Schwinger equations (5) read in Fourier space

$$
\begin{aligned}
& \hat{\boldsymbol{\varepsilon}}(\boldsymbol{\xi})=-\hat{\boldsymbol{\Gamma}}^{0}(\boldsymbol{\xi}): \hat{\boldsymbol{\tau}}(\boldsymbol{\xi})-{ }^{\mathrm{T}} \hat{\boldsymbol{\Upsilon}}^{0}(\boldsymbol{\xi}) \cdot \hat{\boldsymbol{P}}(\boldsymbol{\xi}), \forall \boldsymbol{\xi} \neq 0, \quad \hat{\boldsymbol{\varepsilon}}(0)=\overline{\boldsymbol{\varepsilon}} \\
& \hat{\boldsymbol{E}}(\boldsymbol{\xi})=\hat{\boldsymbol{\Upsilon}}^{0}(\boldsymbol{\xi}): \hat{\boldsymbol{\tau}}(\boldsymbol{\xi})+\hat{\boldsymbol{\Delta}}^{0}(\boldsymbol{\xi}) \cdot \hat{\boldsymbol{P}}(\boldsymbol{\xi}), \forall \boldsymbol{\xi} \neq 0, \quad \hat{\boldsymbol{E}}(0)=\overline{\boldsymbol{E}}
\end{aligned}
$$


The operators $\boldsymbol{\Gamma}^{0}, \boldsymbol{\Delta}^{0}$ and $\boldsymbol{\Upsilon}^{0}$ are explicitly known in Fourier space for whatever anisotropy of the reference piezoelectric medium ${ }^{16,19}$. They are given by

$$
\begin{aligned}
\hat{\Gamma}_{i j k l}^{0}(\boldsymbol{\xi}) & =\frac{1}{4}\left(K_{i l}^{-1} \xi_{k} \xi_{j}+K_{j l}^{-1} \xi_{k} \xi_{i}+K_{i k}^{-1} \xi_{l} \xi_{j}+K_{j k}^{-1} \xi_{i} \xi_{l}\right), \\
\hat{\Upsilon}_{n i j}^{0}(\boldsymbol{\xi}) & =\frac{1}{2}\left(K_{4 j}^{-1} \xi_{i} \xi_{n}+K_{4 i}^{-1} \xi_{j} \xi_{n}\right), \\
\hat{\Delta}_{i j}^{0}(\boldsymbol{\xi}) & =K_{44}^{-1} \xi_{i} \xi_{j},
\end{aligned}
$$

with $\boldsymbol{K}$ a $4 \times 4$ matrix whose components read

$$
\left\{\begin{array}{l}
K_{i j}=C_{p i j q}^{0} \xi_{p} \xi_{q}, \quad \forall(i, j) \in[1,3]^{2} \\
K_{4 i}=K_{i 4}=e_{p i q}^{0} \xi_{p} \xi_{q}, \quad \forall i \in[1,3] \\
K_{44}=-\gamma_{p q}^{0} \xi_{p} \xi_{q} .
\end{array}\right.
$$

When the reference medium does not present piezoelectric coupling (i.e $\left.\boldsymbol{e}^{0}=0\right), \hat{\boldsymbol{\Upsilon}}^{0}=0$ while the operators $\hat{\boldsymbol{\Gamma}}^{0}$ and $\hat{\boldsymbol{\Delta}}^{0}$ reduce, respectively, to the Green operators for elasticity and electrostatics. It is stressed out that the presented formalism can be applied to other coupled-field problems which have the same structure (i.e constitutive laws, divergence and gradient equations). In particular, it can be used to tackle magnetoelectroelastic problems.

\section{FOURIER TRANSFORM BASED METHOD}

\section{A. Iterative scheme}

An efficient numerical scheme based on Fourier transform has been previously proposed to

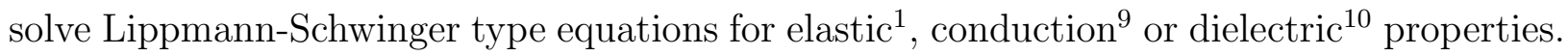
This method uses the fact that the polarization fields are defined locally in real space whereas the Green's operators act locally in Fourier space where they are explicitly known.

The iterative scheme proposed by Moulinec and Suquet for elastic problems ${ }^{1,20}$ can be extended to the context of composite materials with coupled physical properties. In the case of piezoelectric materials, the direct Fourier transform of an initial guess of the polarization fields $\boldsymbol{\tau}$ and $\boldsymbol{P}(6)$, defined on a regular grid, allows to obtain the strain and electric fields in Fourier space. By applying inverse Fourier transform to these latter, the stress and electric induction fields can be computed in real space using the constitutive equations and lead to a new estimate of the polarization fields. The solution fields $\boldsymbol{\varepsilon}$ and $\boldsymbol{E}$ are then obtained by 
successive iterations until convergence criterion is reached. Details on the rate of convergence of the method can be found in Michel et $a l^{21}$.

An attractive feature of this approach is that the pixel discretization of the image of the microstructure can be used to assign the local properties of the material at the nodes of a regular grid. Compared to the FEM, there is thus no need to mesh the microstructure. This allows to consider easily materials with complex microstructures ${ }^{1,6}$. Besides, by using Fast Fourier Transform (FFT) algorithms, the computational cost scales almost linearly with the size of the problem (i.e number of pixels in 2 dimensions and voxels in 3 dimensions).

\section{B. Algorithm}

Periodic microstructures are considered. Consequently, the microstructure of the material is obtained by repeating periodically an elementary pattern (i.e unit-cell). First, this unitcell is discretized in a regular grid and the local properties $\boldsymbol{C}, \boldsymbol{e}$ and $\boldsymbol{\gamma}$ are assigned at each point $\boldsymbol{x}_{g}$ of the grid. Then, the reference medium has to be defined to compute the different Green operators $\left(\boldsymbol{\Gamma}^{0}, \boldsymbol{\Upsilon}^{0}, \boldsymbol{\Delta}^{0}\right)$ in Fourier space at each frequency $\boldsymbol{\xi}_{g}$. The convergence of the method is strongly sensitive to the choice of the reference medium. Based on previous results in the mechanical context ${ }^{1,21}$, we chose

$$
\boldsymbol{C}^{0}=\langle\boldsymbol{C}\rangle, \quad \boldsymbol{e}^{0}=\langle\boldsymbol{e}\rangle \quad \text { and } \quad \gamma^{0}=\langle\gamma\rangle
$$

Following Moulinec and Suquet ${ }^{1}$, the iterative algorithm then reads as follows:

Initialization: $\varepsilon\left(\boldsymbol{x}_{g}\right)=\overline{\boldsymbol{\varepsilon}}, \boldsymbol{E}\left(\boldsymbol{x}_{g}\right)=\overline{\boldsymbol{E}}, \forall \boldsymbol{x}_{g}$

\section{Iterate:}

1. $\boldsymbol{\sigma}\left(\boldsymbol{x}_{g}\right)=\boldsymbol{C}\left(\boldsymbol{x}_{g}\right): \boldsymbol{\varepsilon}\left(\boldsymbol{x}_{g}\right)-{ }^{\mathrm{T}} \boldsymbol{e}\left(\boldsymbol{x}_{g}\right) \cdot \boldsymbol{E}\left(\boldsymbol{x}_{g}\right)$

$\boldsymbol{D}\left(\boldsymbol{x}_{g}\right)=\boldsymbol{e}\left(\boldsymbol{x}_{g}\right): \boldsymbol{\varepsilon}\left(\boldsymbol{x}_{g}\right)+\boldsymbol{\gamma}\left(\boldsymbol{x}_{g}\right) \cdot \boldsymbol{E}\left(\boldsymbol{x}_{g}\right)$

if convergence reached $\longrightarrow$ stop

2. $\boldsymbol{\tau}\left(\boldsymbol{x}_{g}\right)=\boldsymbol{\sigma}\left(\boldsymbol{x}_{g}\right)-\boldsymbol{C}^{0}: \boldsymbol{\varepsilon}\left(\boldsymbol{x}_{g}\right)+{ }^{\mathrm{T}} \boldsymbol{e}^{0} \cdot \boldsymbol{E}\left(\boldsymbol{x}_{g}\right)$

$\boldsymbol{P}\left(\boldsymbol{x}_{g}\right)=\boldsymbol{D}\left(\boldsymbol{x}_{g}\right)-\boldsymbol{e}^{0}: \boldsymbol{\varepsilon}\left(\boldsymbol{x}_{g}\right)-\gamma^{0} \cdot \boldsymbol{E}\left(\boldsymbol{x}_{g}\right)$

3. $\boldsymbol{\tau}\left(\boldsymbol{x}_{g}\right), \boldsymbol{P}\left(\boldsymbol{x}_{g}\right) \stackrel{\mathcal{F}}{\longrightarrow} \hat{\boldsymbol{\tau}}\left(\boldsymbol{\xi}_{g}\right), \hat{\boldsymbol{P}}\left(\boldsymbol{\xi}_{g}\right)$

4. $\hat{\boldsymbol{\varepsilon}}\left(\boldsymbol{\xi}_{g}\right)=-\hat{\boldsymbol{\Gamma}}^{0}\left(\boldsymbol{\xi}_{g}\right): \hat{\boldsymbol{\tau}}\left(\boldsymbol{\xi}_{g}\right)-{ }^{\mathrm{T}} \hat{\boldsymbol{\Upsilon}}^{0}\left(\boldsymbol{\xi}_{g}\right) \cdot \hat{\boldsymbol{P}}\left(\boldsymbol{\xi}_{g}\right), \forall \boldsymbol{\xi}_{g} \neq 0, \quad \hat{\boldsymbol{\varepsilon}}(0)=\overline{\boldsymbol{\varepsilon}}$

$\hat{\boldsymbol{E}}\left(\boldsymbol{\xi}_{g}\right)=\hat{\boldsymbol{\Upsilon}}^{0}\left(\boldsymbol{\xi}_{g}\right): \hat{\boldsymbol{\tau}}\left(\boldsymbol{\xi}_{g}\right)+\hat{\boldsymbol{\Delta}}^{0}\left(\boldsymbol{\xi}_{g}\right) . \hat{\boldsymbol{P}}\left(\boldsymbol{\xi}_{g}\right), \forall \boldsymbol{\xi}_{g} \neq 0, \quad \hat{\boldsymbol{E}}(0)=\overline{\boldsymbol{E}}$ 


$$
\text { 5. } \boldsymbol{\varepsilon}\left(\boldsymbol{x}_{g}\right), \boldsymbol{E}\left(\boldsymbol{x}_{g}\right) \stackrel{\mathcal{F}^{-1}}{\longleftarrow} \hat{\boldsymbol{\varepsilon}}\left(\boldsymbol{\xi}_{g}\right), \hat{\boldsymbol{E}}\left(\boldsymbol{\xi}_{g}\right)
$$

Convergence is reached when the stress $\boldsymbol{\sigma}$ and electric induction $\boldsymbol{D}$ fields are in equilibrium within a certain tolerance error. The convergence criterion is defined as

$$
\max \left(\frac{\left\langle\left\|\boldsymbol{\xi}_{g} \cdot \hat{\boldsymbol{\sigma}}\left(\boldsymbol{\xi}_{g}\right)\right\|^{2}\right\rangle^{\frac{1}{2}}}{\|\hat{\boldsymbol{\sigma}}(0)\|}, \frac{\left\langle\left|\boldsymbol{\xi}_{g} \cdot \hat{\boldsymbol{D}}\left(\boldsymbol{\xi}_{g}\right)\right|\right\rangle^{\frac{1}{2}}}{\|\hat{\boldsymbol{D}}(0)\|}\right) \leq \eta
$$

\section{ILLUSTRATIVE APPLICATIONS}

To assess the proposed numerical scheme, we consider a binary composite made of an isotropic polymer phase (epoxy) and a transversely isotropic piezoelectric ceramics (lead zirconium titanate - PZT) since numerous results exist in the literature. The constitutive law of materials which are transversely isotropic about the $\boldsymbol{e}_{3}$ direction can be written, using Kelvin's notation (see Appendix A), as

$$
\left[\begin{array}{c}
\breve{\sigma}_{1} \\
\breve{\sigma}_{2} \\
\breve{\sigma}_{3} \\
\breve{\sigma}_{4} \\
\breve{\sigma}_{5} \\
\breve{\sigma}_{6} \\
D_{1} \\
D_{2} \\
D_{3}
\end{array}\right]=\left[\begin{array}{ccccccccc}
\breve{C}_{11} & \breve{C}_{12} & \breve{C}_{13} & 0 & 0 & 0 & 0 & 0 & -\breve{e}_{31} \\
\breve{C}_{12} & \breve{C}_{11} & \breve{C}_{13} & 0 & 0 & 0 & 0 & 0 & -\breve{e}_{31} \\
\breve{C}_{13} & \breve{C}_{13} & \breve{C}_{33} & 0 & 0 & 0 & 0 & 0 & -\breve{e}_{33} \\
0 & 0 & 0 & \breve{C}_{44} & 0 & 0 & 0 & -\breve{e}_{15} & 0 \\
0 & 0 & 0 & 0 & \breve{C}_{44} & 0 & -\breve{e}_{15} & 0 & 0 \\
0 & 0 & 0 & 0 & 0 & \breve{C}_{66} & 0 & 0 & 0 \\
0 & 0 & 0 & 0 & \breve{e}_{15} & 0 & \gamma_{11} & 0 & 0 \\
0 & 0 & 0 & \breve{e}_{15} & 0 & 0 & 0 & \gamma_{11} & 0 \\
\breve{e}_{31} & \breve{e}_{31} & \breve{e}_{33} & 0 & 0 & 0 & 0 & 0 & \gamma_{33}
\end{array}\right] \cdot\left[\begin{array}{c}
\breve{\varepsilon}_{1} \\
\breve{\varepsilon}_{2} \\
\breve{\varepsilon}_{3} \\
\breve{\varepsilon}_{4} \\
\breve{\varepsilon}_{5} \\
\breve{\varepsilon}_{6} \\
E_{1} \\
E_{2} \\
E_{3}
\end{array}\right]
$$

The isotropic constitutive law is obtained with the following additional relations

$$
\breve{C}_{33}=\breve{C}_{11}, \breve{C}_{12}=\breve{C}_{13}, \breve{C}_{66}=\breve{C}_{44}, \breve{e}=0, \gamma_{33}=\gamma_{11}
$$

Material data for PZT and epoxy are given in Table II. The FFT-based numerical scheme is now used to compute the response of the binary composite with two different arrangements of the constituents. 
TABLE II: Material properties of the constituents (After Pettermann and Suresh ${ }^{2}$ ). Elastic moduli are in $\mathrm{GPa}$, dielectric moduli are in $\mathrm{nC} / \mathrm{Vm}$ and piezoelectric moduli are in $\mathrm{C} / \mathrm{m}^{2}$.

\begin{tabular}{cccccccccccc}
\hline \hline & $\breve{C}_{11}$ & $\breve{C}_{12}$ & $\breve{C}_{13}$ & $\breve{C}_{33}$ & $\breve{C}_{44} / 2$ & $\breve{C}_{66} / 2$ & $\breve{e}_{31}$ & $\breve{e}_{33}$ & $\breve{e}_{15} / \sqrt{2}$ & $\gamma_{11}$ & $\gamma_{33}$ \\
\hline Epoxy & 8. & 4.4 & 4.4 & 8. & 1.8 & 1.8 & 0. & 0. & 0. & $3.72 \mathrm{e}-02$ & $3.72 \mathrm{e}-02$ \\
PZT & 154.837 & 83.237 & 82.712 & 131.39 & 25.696 & 35.8 & -2.120582 & 9.52183 & 9.34959 & 4.065 & 2.079 \\
\hline \hline
\end{tabular}

\section{A. Laminate composite}

The simplest microstructural case is the laminate composite which is made of succcessive layers of the constituents with arbitrary thickness. Indeed, it is well known that there is an exact solution to the problem and that the fields are constant within each layer. The solution has been given by Benveniste and Dvorak $^{22}$ in the specific case where the axes of transverse isotropy of each layer are parallel to each other and perpendicular to the lamination direction. In the sequel, $\boldsymbol{e}_{3}$ is the axis of transverse isotropy and $\boldsymbol{e}_{2}$ is normal to the layers.

The applied mechanical loading is shear parallel to the layers $\left(\bar{\varepsilon}_{23}\right.$ imposed) and the electric loading is normal to the layers $\left(\bar{E}_{2}\right.$ imposed). The nonvanishing components of the strain $\varepsilon$ and electric field $\boldsymbol{E}$ within layer $(r) \operatorname{read}^{22}$

$$
\begin{aligned}
\varepsilon_{23}^{(r)} & =A_{\varepsilon}^{(r)} \bar{\varepsilon}_{23}+B_{\varepsilon}^{(r)} \bar{E}_{2}, \\
E_{2}^{(r)} & =A_{E}^{(r)} \bar{\varepsilon}_{23}+B_{E}^{(r)} \bar{E}_{2} .
\end{aligned}
$$

For completeness, the expressions of the influence factors $A_{\varepsilon}^{(r)}, B_{\varepsilon}^{(r)}, A_{E}^{(r)}$ and $B_{E}^{(r)}$ are given in Appendix B.

The results of the numerical scheme are presented in Figure 1 for the local strain and electric fields within a binary laminate composite with $c_{1}=c_{2}=0.5$. The unit-cell has been discretized with a regular grid of $32 \times 32$ pixels and the convergence error $\eta$ is equal to $10^{-6}$. Only two iterations are necessary to reach convergence in this case. The FFT computational scheme leads to uniform fields within each layer and the analytical solution (12) is obtained numerically. It is worth noting that convergence is obtained even if the composite present an infinite piezoelectric contrast because of the finite contrast on the elastic and dielectric properties. 

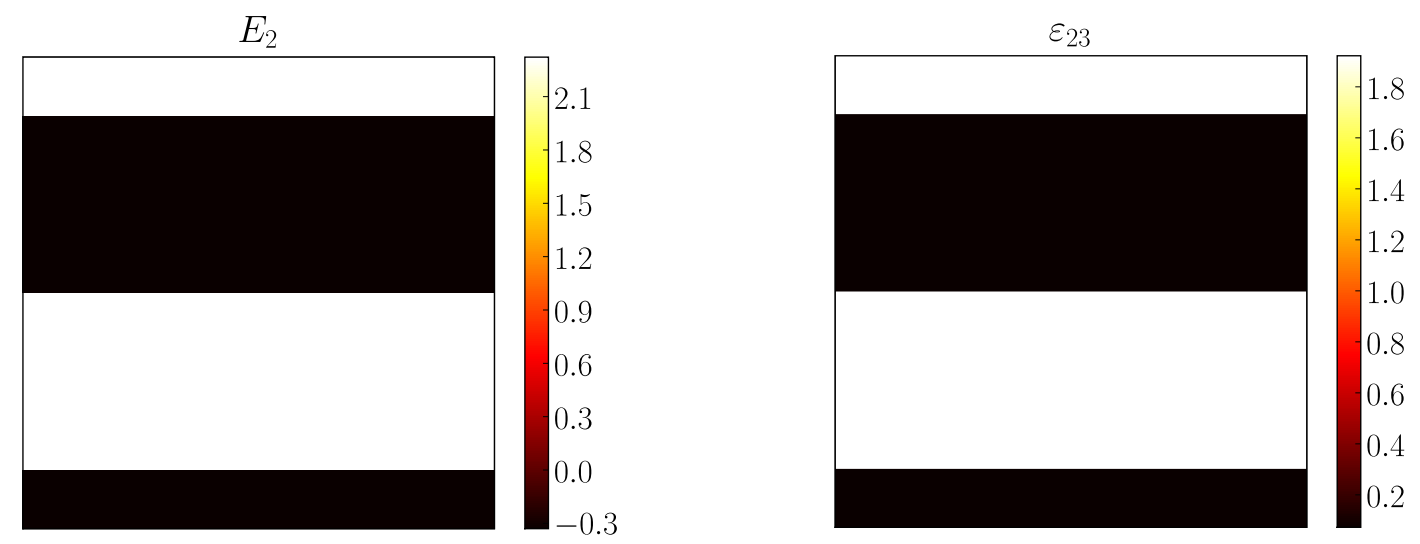

FIG. 1: Component $E_{2}$ of the electric field and component $\varepsilon_{23}$ of the strain field within the twophase laminate composite. Unitary $\bar{E}_{2}$ and $\bar{\varepsilon}_{23}$ imposed.

\section{B. Fiber composite}

We further consider a composite material made of PZT parallel circular fibers embedded in an isotropic epoxy matrix. In the cross section of the material, the fibers are arranged on a periodic square array. A representative unit-cell is thus a square with a fiber at the center and the overall behaviour exhibits quadratic symmetry.

To compute the effective moduli of the composite, the unit-cell has been discretized with a regular grid of $512 \times 512$ pixels and we applied successively six different loadings $\left(\bar{\varepsilon}_{11}\right.$, $\bar{\varepsilon}_{33}, \bar{\varepsilon}_{23}, \bar{\varepsilon}_{12}, \bar{E}_{1}$ and $\bar{E}_{3}$ ). The results obtained with the FFT-based numerical scheme are compared with previously published FEM studies ${ }^{2,3}$ in Table III. For this comparison, note that we converted the overall moduli obtained by FEM which were given at constant electric induction $\boldsymbol{D}$ whereas we used, in the present work, constitutive equations at constant electric field $\boldsymbol{E}$. An excellent agreement is obtained with the FEM results for all effective moduli and the computational method requires, in average, only a few iterations to converge with an error of $10^{-6}$ on the fields equilibrium. Besides, it has been checked that our results fulfill, with a relative error of a few percents, the universal relations for the overall moduli of fiber composite materials with quadratic (tetragonal) symmetry ${ }^{22}$. For illustrative purpose, the distribution of the local fields $E_{1}$ and $\varepsilon_{13}$ for a macroscopic loading $\bar{E}_{1}$ are plotted in Figure 2. Almost uniform fields are predicted within the fibers where, as expected, a low electrical field takes place. Significant longitudinal shear strain is predicted in the matrix at 

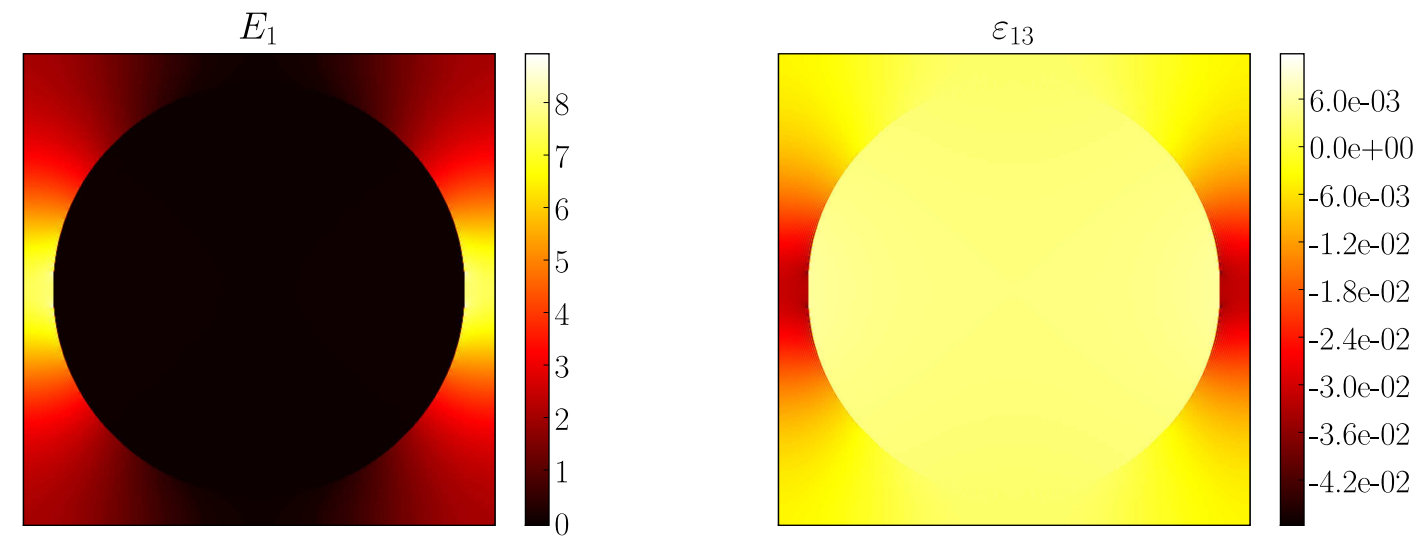

FIG. 2: Component $E_{1}$ of the electric field and component $\varepsilon_{13}$ of the strain field within the fiber composite with square arrangement for an unitary uniaxial applied electric field $\bar{E}_{1}$.

the fiber-matrix interface normal to the $\boldsymbol{e}_{1}$ axis.

TABLE III: Comparison of FFT and FEM results ${ }^{2,3}$ for the effective moduli of the fibrous composite with $60 \%$ volume fraction of fibers. Units are the same as Table II.

\begin{tabular}{lccr}
\hline \hline & FFT & FEM $^{2}$ & FEM $^{3}$ \\
\hline$\breve{C}_{11}$ & 25.11 & 25.16 & 25.14 \\
$\breve{C}_{12}$ & 8.57 & 8.73 & 8.68 \\
$\breve{C}_{13}$ & 11.78 & 11.85 & 11.83 \\
$\breve{C}_{33}$ & 54.46 & 54.61 & 54.52 \\
$\breve{C}_{44} / 2$ & 6.63 & 6.68 & 6.64 \\
$\breve{C}_{66} / 2$ & 4.62 & 4.64 & 4.64 \\
$\breve{e}_{31}$ & -0.20 & -0.20 & -0.20 \\
$\breve{e}_{33}$ & 6.43 & 6.45 & 6.45 \\
$\breve{e}_{15} / \sqrt{2}$ & 0.052 & 0.052 & 0.052 \\
$\gamma_{11}$ & 0.157 & 0.158 & 0.157 \\
$\gamma_{33}$ & 1.277 & 1.282 & 1.280 \\
\hline \hline
\end{tabular}




\section{CONCLUDING REMARKS}

By following the approach initially developed for uncoupled mechanical constitutive behaviour by Moulinec and Suquet ${ }^{1}$, we have presented an original computational scheme to predict the local and overall responses of heterogeneous electroelastic materials. This scheme, which makes use of fast Fourier transform algorithms and allows to use the image of the material as direct input, is a viable alternative to finite-element analysis to tackle the response of piezoelectric composites. In this article, the method has been successfully compared to analytical solutions and finite-element results for simple binary composites. However, the method is particularly well suited to consider heterogeneous materials with complex microstructures like polycrystalline ceramics. Such microstructural computations have been previously performed in the uncoupled mechanical context ${ }^{6,8}$. Eventually, it is noted that this computational procedure can be used for nonlinear heterogeneous materials with coupled constitutive equations and is not restricted to the coupled piezoelectric behaviour. It is relevant for other "multiphysics" problems which adopt a similar mathematical description (e.g magnetoelectroelasticity). 


\section{Appendix A: Kelvin notation for symmetric tensors}

The Kelvin notation ${ }^{23}$, initially introduced in elasticity, can be advantageously used to write the electroelastic constitutive equations as

$$
\left\{\begin{array}{l}
\breve{\sigma}_{\alpha}=\breve{C}_{\alpha \beta} \breve{\varepsilon}_{\beta}-{ }^{\mathrm{T}} \breve{e}_{\alpha j} E_{j} \\
D_{i}=\breve{e}_{i \beta} \breve{\varepsilon}_{\beta}+\gamma_{i j} E_{j}
\end{array}\right.
$$

where $(\alpha, \beta) \in[1,6]^{2}$ and $(i, j) \in[1,3]^{2}$. In the orthonormal basis $\left(\boldsymbol{e}_{i}, i \in[1,3]\right)$, the components of $\breve{\boldsymbol{\sigma}}, \breve{\varepsilon}, \breve{\boldsymbol{C}}$ and $\breve{\boldsymbol{e}}$ are

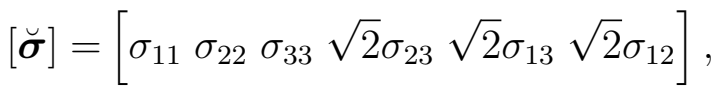

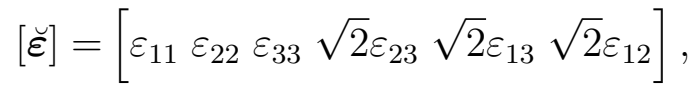

$$
\begin{aligned}
& {[\breve{\boldsymbol{C}}]=\left[\begin{array}{cccccc}
C_{1111} & C_{1122} & C_{1133} & \sqrt{2} C_{1132} & \sqrt{2} C_{1131} & \sqrt{2} C_{1121} \\
C_{2211} & C_{2222} & C_{2233} & \sqrt{2} C_{2232} & \sqrt{2} C_{2231} & \sqrt{2} C_{2221} \\
C_{3311} & C_{3322} & C_{3333} & \sqrt{2} C_{3332} & \sqrt{2} C_{3331} & \sqrt{2} C_{3321} \\
\sqrt{2} C_{2311} & \sqrt{2} C_{2322} & \sqrt{2} C_{2333} & 2 C_{2332} & 2 C_{2331} & 2 C_{2321} \\
\sqrt{2} C_{1311} & \sqrt{2} C_{1322} & \sqrt{2} C_{1333} & 2 C_{1332} & 2 C_{1331} & 2 C_{1321} \\
\sqrt{2} C_{1211} & \sqrt{2} C_{1222} & \sqrt{2} C_{1233} & 2 C_{1232} & 2 C_{1231} & 2 C_{1221}
\end{array}\right]} \\
& {[\breve{\boldsymbol{e}}]=\left[\begin{array}{cccccc}
e_{111} & e_{122} & e_{133} & \sqrt{2} e_{123} & \sqrt{2} e_{113} & \sqrt{2} e_{112} \\
e_{211} & e_{222} & e_{233} & \sqrt{2} e_{223} & \sqrt{2} e_{213} & \sqrt{2} e_{212} \\
e_{311} & e_{322} & e_{333} & \sqrt{2} e_{323} & \sqrt{2} e_{313} & \sqrt{2} e_{312}
\end{array}\right] .}
\end{aligned}
$$

The coupled constitutive equations can thus be expressed in the following concise form

$$
J_{\alpha}=L_{\alpha \beta} F_{\beta}, \quad(\alpha, \beta) \in[1,9]^{2},
$$


with

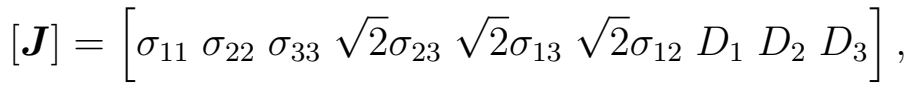

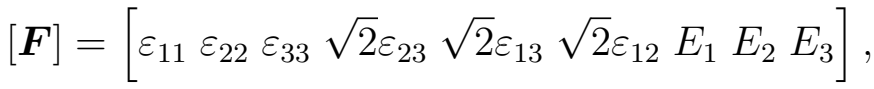

$$
\begin{aligned}
& {[\boldsymbol{L}]=\left[\begin{array}{ccccccccc}
C_{1111} & C_{1122} & C_{1133} & \sqrt{2} C_{1132} & \sqrt{2} C_{1131} & \sqrt{2} C_{1121} & -e_{111} & -e_{211} & -e_{311} \\
C_{2211} & C_{2222} & C_{2233} & \sqrt{2} C_{2232} & \sqrt{2} C_{2231} & \sqrt{2} C_{2221} & -e_{122} & -e_{222} & -e_{322} \\
C_{3311} & C_{3322} & C_{3333} & \sqrt{2} C_{3332} & \sqrt{2} C_{3331} & \sqrt{2} C_{3321} & -e_{133} & -e_{233} & -e_{333} \\
\sqrt{2} C_{2311} & \sqrt{2} C_{2322} & \sqrt{2} C_{2333} & 2 C_{2332} & 2 C_{2331} & 2 C_{2321} & -\sqrt{2} e_{123} & -\sqrt{2} e_{223} & -\sqrt{2} e_{323} \\
\sqrt{2} C_{1311} & \sqrt{2} C_{1322} & \sqrt{2} C_{1333} & 2 C_{1332} & 2 C_{1331} & 2 C_{1321} & -\sqrt{2} e_{113} & -\sqrt{2} e_{213} & -\sqrt{2} e_{313} \\
\sqrt{2} C_{1211} & \sqrt{2} C_{1222} & \sqrt{2} C_{1233} & 2 C_{1232} & 2 C_{1231} & 2 C_{1221} & -\sqrt{2} e_{112} & -\sqrt{2} e_{212} & -\sqrt{2} e_{312} \\
e_{111} & e_{122} & e_{133} & \sqrt{2} e_{123} & \sqrt{2} e_{113} & \sqrt{2} e_{112} & \gamma_{11} & \gamma_{12} & \gamma_{13} \\
e_{211} & e_{222} & e_{233} & \sqrt{2} e_{223} & \sqrt{2} e_{213} & \sqrt{2} e_{212} & \gamma_{12} & \gamma_{22} & \gamma_{23} \\
e_{311} & e_{322} & e_{333} & \sqrt{2} e_{323} & \sqrt{2} e_{313} & \sqrt{2} e_{312} & \gamma_{13} & \gamma_{23} & \gamma_{33}
\end{array}\right] .}
\end{aligned}
$$

\section{Appendix B: Expression of the influence factors for the binary laminate composite}

Analytic expressions of the influence factors of relation (12) can be found $\mathrm{in}^{22}$. They read, for phase (1),

$$
\begin{aligned}
A_{\varepsilon}^{(1)} & =\left[\left(c_{1} \gamma_{11}^{(2)}+c_{2} \gamma_{11}^{(1)}\right) \breve{C}_{44}^{(2)}+\left(c_{1} \breve{e}_{15}^{(2)}+c_{2} \breve{e}_{15}^{(1)}\right) \breve{e}_{15}^{(2)}\right] / 2 D, \\
B_{\varepsilon}^{(1)} & =c_{2}\left(\gamma_{11}^{(2)} \breve{e}_{15}^{(1)}-\gamma_{11}^{(1)} \breve{e}_{15}^{(2)}\right) / 2 D \sqrt{2}, \\
A_{E}^{(1)} & =c_{2}\left(\breve{C}_{44}^{(1)} \breve{e}_{15}^{(2)}-\breve{C}_{44}^{(2)} \breve{e}_{15}^{(1)}\right) / D \sqrt{2}, \\
B_{E}^{(1)} & =\left[\breve{e}_{15}^{(2)}\left(c_{1} \breve{e}_{15}^{(2)}+c_{2} \breve{e}_{15}^{(1)}\right)+\gamma_{11}^{(2)}\left(c_{1} \breve{C}_{44}^{(2)}+c_{2} \breve{C}_{44}^{(1)}\right)\right] / 2 D, \\
D & =\frac{1}{2}\left[\left(c_{1} \breve{e}_{15}^{(2)}+c_{2} \breve{e}_{15}^{(1)}\right)^{2}+\left(c_{1} \breve{C}_{44}^{(2)}+c_{2} \breve{C}_{44}^{(1)}\right)\left(c_{1} \gamma_{11}^{(2)}+c_{2} \gamma_{11}^{(1)}\right)\right] .
\end{aligned}
$$

$c_{1}$ and $c_{2}$ are the volumic fractions of each phase. Corresponding influence factors for phase (2) are simply obtained by interchanging indices $(1) \leftrightarrow(2)$.

* rb@lpmtm.univ-paris13.fr

1 H. Moulinec and P. Suquet, Comput. Methods Appl. Mech. Engrg. 157, 69 (1998).

2 H. E. Pettermann and S. Suresh, Int. J. Solids Struct. 37, 5447 (2000). 
3 H. Berger, S. Kari, U. Gabbert, R. Rodriguez-Ramos, J. Bravo-Castillero, R. Guinovart-Diaz, F. J. Sabina, and G. A. Maugin, Smart Mater. Struct. 15, 451 (2006).

4 Y. Uetsuji, Y. Nakamura, S. Ueda, and E. Nakamachi, Modelling Simul. Mater. Sci. Eng. 12, S303 (2004).

5 J. Lee, J. G. B. IV, and D. C. Lagoudas, International Journal of Engineering Science 43, 790 (2005).

${ }^{6}$ R. A. Lebensohn, R. Brenner, O. Castelnau, and A. D. Rollett, Acta Mater. 56, 3914 (2008).

7 R. A. Lebensohn, Acta Mater. 49, 2723 (2001).

8 R. Brenner, R. A. Lebensohn, and O. Castelnau, Int. J. Solids Struct., in press.

9 D. J. Eyre and G. W. Milton, Journal of Physique III 6, 41 (1999).

10 H. Ma, B. Zhang, W. Y. Tam, and P. Sheng, Physical Review B 61, 962 (2000).

11 P. Suquet, in Homogenization Techniques for Composite Media, edited by E. Sanchez-Palencia and A. Zaoui (Springer-Verlag, 1987), pp. 194-278.

12 D. M. Barnett and J. Lothe, Phys. Stat. Sol. b 67, 105 (1975).

13 R. Zeller and P. H. Dederichs, Phys. Status Solidi (b) 55, 831 (1973).

14 E. Kröner and H. Koch, Solid Mechanics Archives 1, 183 (1976).

15 W. F. Deeg, Ph.D. thesis, Stanford University, U.S.A (1980).

16 B. Wang, Int. J. Solids Struct. 29, 293 (1992).

17 M. L. Dunn and M. Taya, Proc. R. Soc. Lond. 443, 265 (1993).

18 J. H. Huang and W.-S. Kuo, Acta Mater. 44, 4889 (1996).

19 V. M.Levin, M. I.Rakovskaja, and W. S. Kreher, Int. J. Solids Struct. 25, 1572 (1999).

20 H. Moulinec and P. Suquet, C. R. Acad. Sci. Paris 318, 1417 (1994).

21 J. C. Michel, H. Moulinec, and P. Suquet, Int. J. Numer. Meth. Engng 52, 139 (2001).

22 Y. Benveniste and G. J. Dvorak, J. Mech. Phys. Solids 40, 1295 (1992).

23 M. M. Mehrabadi and S. C. Cowin, Quart. J. Mech. Appl. Math. 43, 15 (1990). 